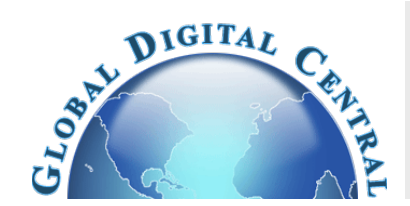

\title{
INFLUENCE OF CRITICAL PARAMETERS OF THERMOPHOROSIS ON MHD NON-DARCY FLOW OF A CASSON FLUID PAST A PERMEABLE STRETCHING SHEET
}

\author{
Kolli Vijaya ${ }^{a,}{ }^{*}$, G. Venkata Ramana Reddy ${ }^{a}$ \\ ${ }^{a}$ Research Scholar, Koneru Lakshmaiah Education Foundation, Vaddeswaram, Guntur, India-522502. \\ ${ }^{a}$ Department of Mathematics, Koneru Lakshmaiah Education Foundation, Vaddeswaram, Guntur, India-522502.
}

\begin{abstract}
Present investigation aims at analyzing the properties of heat and transport phenomena of thermal energy and species mass in a non-Darcy Casson fluid flow induced by a erected porous elongated surface considering the effect of non-linear radioactive heat, thermophorosis, first order chemical reaction, Newtonian heating, thermo diffusion, permeability and slip conditions. Influence of critical parameters are widely studied. By inducing the variables of similarity the basic boundary layer equations are transmuted into dimensionless equations and are resolved arithmetically using Runge Kutta - Fehlberg shooting techniques method. The dominance of critical parameters against velocity, temperature, and concentration are explicated through graphs. Non Newtonian nature of Casson fluid is detected by observing the influence of Casson parameter on velocity, temperature, and concentration profiles. The numerical values of Skin friction coefficient, Nusselt number, and Sherwood number for different parameters are calculated and presented in tabular form.
\end{abstract}

Key words: $M H D$, Casson fluid, non-linear thermal radiation, non-Darcy porous medium, viscous dissipation, Electric field parameter.

\section{INTRODUCTION}

Transport phenomena of fluids through different geometric porous media at high Reynolds number is attracting the attention of both technologists, as well as biologists because of its application in hydrology, petroleum engineering, marine engineering, chemical engineering, bio fluids and so forth. When the fluid flow takes place through a porous medium at high Reynolds number, inertia effect is dominant which leads the flow to nonDarcy nature. More over study of porous media theories are important and is helpful to have deep insight of fine grained, muddy sediments de nitrification rate measurements of continental shelves which have been made with free grained, muddy sediments. Moreover fluid flows through natural and artificial porous media plays an important role in biological systems like flow patterns in plants through xylem and phloem, as well a central nervous system of animals. Narayana et al. (2013) gave elaborate solution and explained the non- Darcy nature of non- Newtonian power law liquid using semi numerical successive linearization method. Chamkha (1997) in his study observed that ambient temperature is an increasing function with the distance along the plate. Zeeshan et al. (2016) examined the non-Darcy boundary layer flow of non-conducting viscous fluid with magnetic Ferro particles over a permeable linearly stretching surface with ohmic dissipation and mixed convective heat transfer. By maintaining the fixed temperature and concentration Partha (2009) examined the impact of Soret and Dufour on thermophoresis particle deposition on the wall in the existence of suction and injection. Vempati and Laxmi-Narayana-Gari (2010) analyzed MHD flow under different conditions. Dulal Pal et al. (2012) studied heat and mass transfer characteristic of the flow over a stretching surface through a porous medium considering Soret and Dofour effects in the presence of electrical field and non-uniform heat source/sink. The properties of nonNewtonian fluid were studied by many mathematical investigators for the reason that their exhaustive applications in numerous sectors like chemical industries, metallurgy, astro physics, and geology and food industry. Shampoos, tomato sauce, gels, paste, colloidal suspensions demonstrates non -Newtonian behavior. The rheological properties of such fluids play an imperative role in lubricant oils, greases and in coating processes. In several such applications, the ratio between the shear stress versus the shear rate is extremely non-linear and the ratio between shear stress and shear rate is no more a continuous at any point of time. Many fluids existing in nature are non-linear. Several other categories of non-Newtonian fluids are visco-elastic, micro polar, and Casson fluid. Casson fluid can be defined as a shear thinning fluid which is assumed to have an infinite viscosity at zero shear rate and yield stress below which no flow takes place. Pavlov (1974) was the pioneer who discussed the influence of magnetic field on MHD flow past a stretching surface. Andersson (1992) gave brief description on viscoelastic fluid past a stretching surface. Swathi Mukopadhyaya et al. (2013) investigated the flow of a Casson fluid over an unsteady stretching permeable surface with first order destructive chemical reaction. Their numerical results reveal that the velocity and the corresponding boundary layer thickness are decreasing functions of the Casson parameter. Furthermore, the heat and mass transfer analysis for MHD Casson fluid boundary layer flow over a permeable stretching sheet through a porous medium is carried out by Gireesha et al. (2015). Ramana Reddy et al. (2016a, 2016b) explored different types of flows over a vertical surface in porous media. Khan (2019) in his study analyzed Nano material flows between two stretchable rotating discs. Vijaya et al. (2018) gave systematic study of Soret and radiation effects on an unsteady flow of a Casson fluid through porous vertical channel with contractions. MHD

*Corresponding author, Email: vijiji.maths@gmail.com 
flow of Carreau Nono fluid explored using CNT over a nonlinear stretching sheet is studied by Nagalakshmi et al. (2020). Kolli Vijaya et al. (2019) gave brief study on Casson fluid Flow Over vertical Porous Plate in the presence of Radiation, Soret and Chemical reaction. Rohmat Ellahi et al. (2019) gave mathematical modelling and studied Nano fluid entropy generation. Reddy et al. (2015) examined chemical reaction effects on MHD free convection dissipation fluid flow past an inclined porous surface.

Motivated by the above mentioned studies in the present study we investigate the effect of non - linear thermal radiation and velocity slip on the MHD non-Darcy flow of an incompressible, electrically conducting Casson fluid past a permeable stretching sheet taking joule heating and thermophoresis into account. Non Darcy nature of the Casson fluid for different critical parameters is observed for both suction and injection.

\section{MATHEMATICAL FORMULATION}

The steady non-Darcy mixed convective transport phenomena of an incompressible and electrically conducting Casson fluid flow along vertical elongated sheet implanted in a porous medium is reflected in this study. The frame of reference for this problem is taken along x-axis, in which the sheet is elongated and y - axis is perpendicular to it as exposed in Fig. 1. The region of the flow is subjected to a transverse magnetic field $\vec{B}=\left(0, B_{0}, 0\right)$ of uniform intensity, and uniform electric field $\vec{E}=$ $\left(0, E_{0}, 0\right)$. By applying two equal and opposite forces on the elastic boundary sheet the flow is generated which is linear. The Maxwell's equations are $\nabla \cdot \vec{B}=0$ and $\nabla X \bar{E}=0$. From Ohm's law $\vec{J}=\sigma(\vec{E}+\vec{q} \mathrm{X}$ $\vec{B}$ ) where $\vec{J}$ is the Joule current, $\sigma$ is the magnetic permeability and $\vec{q}$ is the fluid velocity. The magnetic field produced due to the motion of the electric field is very small and it can be neglected.

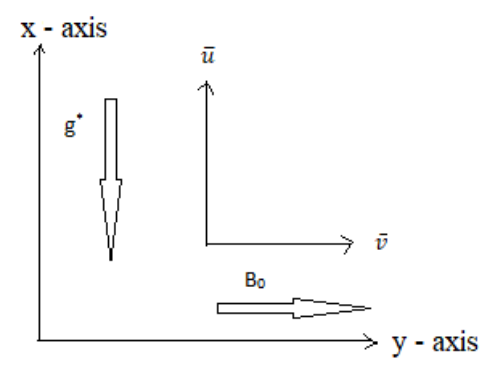

Fig. 1 Geometrical outline of the flow

Casson fluid is Non Newtonian in nature and its constitutive equations (Eldabe and Salwa, (1995)) are written as

$\tau_{i j}=\left\{\begin{array}{l}2 \mathrm{e}_{\mathrm{ij}}\left(\mu_{\mathrm{B}}+\frac{\mathrm{P}_{\mathrm{y}}}{\sqrt{2 \pi}}\right), \pi>\pi_{\mathrm{c}}, \\ 2 \mathrm{e}_{\mathrm{ij}}\left(\mu_{\mathrm{B}}+\frac{\mathrm{P}_{\mathrm{y}}}{\sqrt{2 \pi_{\mathrm{c}}}}\right), \pi<\pi_{\mathrm{c}}\end{array}\right.$

Where $\tau_{i j}$ is the $(i, j)^{\text {th }}$ stress tensor component. $\mu_{B}$ is the plastic dynamic viscosity of the non-Newtonian fluid, $P_{y}$ is the yield stress of the fluid, $\pi$ is the product of the component of deformation rate with itself, namely, $\pi=e_{i j} e_{i j}$, and $e_{i j}$ is the $(i, j)^{t h}$ component of deformation rate, and $\pi_{c}$ is critical value of $\pi$ based on non-Newtonian model. The governing equations with reference to the symbols in nomenclature are given as follows.

$$
\frac{\partial \bar{u}}{\partial x}+\frac{\partial \bar{v}}{\partial y}=0
$$

$$
\begin{aligned}
& \frac{1}{\varepsilon^{2}}\left(\bar{u} \frac{\partial \bar{u}}{\partial x}+\bar{v} \frac{\partial \bar{u}}{\partial y}\right)=\frac{v}{\varepsilon}\left(1+\frac{1}{\gamma}\right) \frac{\partial^{2} \bar{u}}{\partial y^{2}}+\frac{\sigma}{\rho}\left(E_{0} B_{0}-B_{0}^{2} \bar{u}\right) \\
& -\frac{v}{k_{1}} \bar{u}-\frac{C_{b}}{\sqrt{k_{1}}} \bar{u}^{2}+g^{*} \beta_{T}\left(T^{*}-T_{\infty}\right)+g^{*} \beta_{C}\left(C^{*}-C_{\infty}\right) \\
& \left(\bar{u} \frac{\partial T^{*}}{\partial x}+\bar{v} \frac{\partial T^{*}}{\partial y}\right)=\frac{k}{\rho c_{p}} \frac{\partial^{2} T^{*}}{\partial y^{2}}+\frac{16 \sigma^{*}}{3 k^{*} \rho c_{p}} \frac{\partial}{\partial y}\left(T^{* 3} \frac{\partial T^{*}}{\partial y}\right)+ \\
& \frac{\mu}{\rho c_{p}}\left(1+\frac{1}{\gamma}\right)\left(\frac{\partial \bar{u}}{\partial y}\right)^{2}+\frac{\sigma}{\rho c_{p}}\left(\bar{u} B_{0}-E_{0}\right)^{2} \\
& \left(\bar{u} \frac{\partial C^{*}}{\partial x}+\bar{v} \frac{\partial C^{*}}{\partial y}\right)=D \frac{\partial^{2} C^{*}}{\partial y^{2}}+\frac{D k_{T^{*}}}{T_{m}} \frac{\partial^{2} T^{*}}{\partial y^{2}}-k_{0}\left(C^{*}-C_{\infty}\right) \\
& -\frac{\partial}{\partial y}\left(V_{T^{*}}\left(C^{*}-C_{\infty}\right)\right.
\end{aligned}
$$

The boundary conditions of the problem are

$$
\begin{aligned}
& \bar{u}=\bar{u}_{w}(x)+N_{1}\left(1+\frac{1}{\gamma}\right) v \frac{\partial u}{\partial y} \quad, \quad \bar{v}=\bar{v}_{w}, \quad T^{*}=T_{w}=T_{\infty}+ \\
& A_{0}\left(\frac{x}{L}\right)^{2}, \quad C^{*}=C_{w}=C_{\infty}+A_{1}\left(\frac{x}{L}\right)^{2} \text { at } y=0 \\
& \bar{u} \rightarrow 0, \quad T^{*} \rightarrow T_{\infty}, \quad C^{*} \rightarrow C_{\infty} \quad \text { as } y \rightarrow \infty
\end{aligned}
$$

where $\bar{u}_{w}(x)=a x$ is the velocity of the elongating surface, $a$ is the proportionality constant, $N_{1}$ is the velocity slip factor , $\bar{v}_{w}=$ $-\left(v \bar{u}_{\mathrm{w}} / x\right)^{1 / 2} \mathrm{fw}$ is the mass transmission speed on the surface of the sheet with $f_{w}>0$ for injection (blowing) and $f_{w}<0$ for suction, and $f_{w}=0$ corresponds to an impervious sheet, $T_{w}$ is the uniform temperature and $T_{\infty}$ is the fluid temperature when $T_{w}>T_{\infty}, C_{w}$ is the uniform concentration and $C_{\infty}$ is the fluid concentration in the free stream with $C_{w}>C_{\infty}$.In the above equation (5) with reference to Talbot et al (1980) $V_{T^{*}}$ is taken as the thermophoresis velocity as $V_{T^{*}}=$ $-\left(\frac{k_{t} v}{T_{r e f}}\right)\left(\frac{\partial T^{*}}{\partial y}\right)$, with $k_{t} v$ as thermophoretic diffusivity and $T_{r e f}$ as some reference temperature.

\section{METHOD OF SOLUTION}

Subsequent likeness variables are introduced to convert partial differential equations (2) to equation (5) into ordinary differential equations.

$\bar{u}=a x f^{\prime}(\eta), \bar{v}=-\sqrt{a v} f(\eta), \eta=\sqrt{\frac{a}{v}} y$,
$\theta(\eta)=\frac{T-T_{\infty}}{T_{w}-T_{\infty}}, \quad \phi(\eta)=\frac{C-C_{\infty}}{C_{w}-C_{\infty}}$,

Substituting the similarity variables defined in equation (7) into the governing equations (2) - equations (5) along with the boundary conditions, the following dimensionless equations were derived.

$\left(1+\frac{1}{\gamma}\right) \frac{f^{\prime \prime \prime}}{\varepsilon}+\frac{f f^{\prime \prime}}{\varepsilon^{2}}-\frac{f^{\prime 2}}{\varepsilon^{2}}+M^{2}\left(\zeta-f^{\prime}\right)-\xi f^{\prime 2}-k_{p} f^{\prime}$

$+\lambda(\theta+N \phi)=0$

$\theta^{\prime \prime}+N r\left[\left(1+\left(\theta_{w}-1\right) \theta\right)^{3}\right] \theta^{\prime \prime}+3 N r\left[\left(\theta_{w}-1\right)\left(1+\left(\theta_{w}-1\right) \theta\right)^{2}\right] \theta^{\prime 2}+$

$\operatorname{Pr}\left[f \theta^{\prime}-2 f^{\prime} \theta+M^{2} E c\left(\zeta-f^{\prime}\right)^{2}+E c\left(1+\frac{1}{\gamma}\right)\left(f^{\prime \prime}\right)^{2}\right]=0$

$\phi^{\prime \prime}+\operatorname{Sc}\left(f \phi^{\prime}-2 f^{\prime} \phi-\chi \phi+\operatorname{Sr} \theta^{\prime \prime}-\tau\left(\theta^{\prime} \phi^{\prime}+\phi \theta^{\prime \prime}\right)\right)=0$

The boundary conditions (6) become 


$$
\begin{aligned}
& f(0)=f w, f^{\prime}(0)=1+\mathrm{h}\left(1+\frac{1}{\gamma}\right) \mathrm{f}^{\prime \prime}(0) \\
& \theta(0)=1, \phi(0)=1 \text { at } \eta=0, \\
& f^{\prime}(\infty)=0, \theta(\infty)=0, \phi(\infty)=0 \text { as } \eta \rightarrow \infty,
\end{aligned}
$$

where the dimensionless critical parameters are as follows.

$$
\begin{aligned}
& \gamma=\frac{\mu_{B} \sqrt{2 \pi_{c}}}{p_{y}} \\
& \mathrm{M}^{2}=\frac{\sigma \mathrm{B}_{0}^{2}}{\rho \mathrm{a}} \\
& \zeta=\frac{E_{0}}{B_{0} a x} \\
& \xi=\frac{C_{b} x}{\sqrt{k_{1}}} \\
& k_{p}=\frac{v}{k_{1} a} \\
& \lambda=\frac{G r_{x}}{R e_{x}^{2}} \\
& N=\frac{G r_{x}}{G r_{x}^{*}} \\
& N r=\frac{16 \sigma^{*} T_{\infty}^{3}}{3 k k^{*}} \\
& \theta_{w}=\frac{\mathrm{T}_{\mathrm{w}}}{\mathrm{T}_{\infty}} \\
& \operatorname{Pr}=\frac{\rho c_{p} v}{k} \\
& E c=\frac{a L^{2}}{c_{p} A_{0}} \\
& S c=\frac{v}{D} \\
& \chi=\frac{k_{0}}{a} \\
& S r=\frac{D k_{T}\left(T_{w}-T_{\infty}\right)}{v T_{m}\left(C_{w}-C_{\infty}\right)} \\
& \tau=\frac{-k_{T}\left(T_{w}-T_{\infty}\right)}{T_{r e f}} \\
& G r_{x}=\frac{g \beta_{T}\left(T^{*}-T_{\infty}\right) x^{3}}{v^{2}} \\
& G r_{x}^{*}=\frac{g \beta_{C}\left(C^{*}-C_{\infty}\right) x^{3}}{v^{2}} \\
& h=N_{1} \sqrt{a v}
\end{aligned}
$$

(Casson parameter)

(Magnetic field parameter)

(Electric field parameter)

(Inertial parameter)

(Porous parameter)

(Thermal Buoyancy parameter)

(Solutal buoyancy parameter)

(Thermal radiation parameter)

(Temperature ratio parameter)

(Prandtl number)

(Eckert number)

(Schmidt number)

(Chemical reaction parameter)

(Soret number)

(Thermophorotic parameter)

(Grashof number due to temperature)

(Grashof number due to Concentration)

(Velocity slip parameter)

The significant physical quantities of engineering interest in this problem are the skin friction coefficient $C_{f}$, local Nusselt number $N u_{x}$ and local Sherwood number $S h_{x}$ which are defined as follows:

$C_{f}=\frac{2 \tau_{w}}{\rho u_{w}^{2}}, N u_{x}=\frac{x q_{w}}{K\left(T_{w}-T_{\infty}\right)}, S h_{x}=\frac{x q_{m}}{D_{m}\left(C_{w}-C_{\infty}\right)}$

Here the wall shear stress $\tau_{w}$, heat transfer $q_{w}$ and mass transfer $q_{m}$ are given by

$\tau_{w}=\mu\left(1+\frac{1}{\gamma}\right)\left(\frac{\partial u}{\partial y}\right)_{y=0}, q_{w}=-K\left(\frac{\partial T}{\partial y}\right)_{y=0}$ and $q_{m}=-D\left(\frac{\partial C}{\partial y}\right)_{y=0}$

Making use the variables in equation (7), using equation (14) in equation (13) we obtain

$$
\begin{aligned}
& \frac{1}{2} C_{f} \sqrt{R e_{x}}=\left(1+\frac{1}{\beta}\right) f^{\prime \prime}(0) \\
& N u_{x} / R e_{x}^{1 / 2}=-\left(1+N r \theta_{w}^{3}\right) \theta^{\prime}(0) \\
& S h_{x} / R e_{x}^{1 / 2}=-\phi^{\prime}(0)
\end{aligned}
$$

where $R e_{x}=\frac{u_{w} x}{v}$ is the local Reynolds number.

Equations (8) to equations (10) together with the boundary conditions are solved numerically using the Runge-Kutta-Fehlberg method along with shooting technique.

To validate the numerical code, comparison of results of the present analysis is made with the published results and is presented in Table. 1 and Table. 2 as a limiting case. Table .1 shows comparison of $-f^{\prime \prime}(0)$ with those obtained by Hayat et al. (2009), Mabood and Das (2016), Mabood et al. (2016) in the absence of $\zeta=\xi=\lambda=N$ $=k_{p}=f w=h=\mathrm{Nr}=\mathrm{Ec}=\mathrm{Sc}=\chi=\mathrm{Sr}=\tau=0$ and $\theta_{w}=\varepsilon=1.0$ in the case of Newtonian fluid for different values of M. Table. 2 presents the comparison of $-\theta^{\prime}(0)$ for different values of Prandtl number with those of Chen (1998), Grubka and Bobba (1985), Dulal Pal and Mondal (2012). These two tables show that the results of the current investigation are in very good agreement with previously published results. Table. 3 gives the numerical values of Skin friction coefficient, Nusselt number and Sherwood number for various values of pertinent parameters.

Table .1: Comparison values of $-\boldsymbol{f}^{\prime \prime}(\mathbf{0})$ for various values of $\mathrm{M}$ when $\zeta=\xi=\lambda=N=k_{p}=f w=h=\mathrm{Nr}=\mathrm{Ec}=\mathrm{Sc}=\chi=\mathrm{Sr}=\tau=0$

\begin{tabular}{|l|c|c|c|c|}
\multicolumn{5}{|c|}{ and $\theta_{w}=\varepsilon=1.0$ and $\boldsymbol{\gamma} \rightarrow \infty$} \\
\hline \multicolumn{1}{|c|}{$\mathbf{M}$} & $\begin{array}{c}\text { Hayat } \text { et al. } \\
(\mathbf{2 0 0 9 )}\end{array}$ & $\begin{array}{c}\text { Mabood and } \\
\text { Das (2016) }\end{array}$ & $\begin{array}{c}\text { Mabood } \text { et } \\
\text { al. (2016) }\end{array}$ & $\begin{array}{c}\text { Present } \\
\text { results }\end{array}$ \\
\hline & & & & \\
0 & -1.00000 & -1.000008 & -1.0000084 & -1.00000 \\
1 & 1.4142 & 1.4142135 & 1.41421356 & 1.41421 \\
5 & 2.4494 & 2.4494897 & 2.44948974 & 2.44949 \\
10 & 3.31662 & 3.31662 & 3.316624 & 3.31662 \\
50 & 7.14142 & 7.1414284 & 7.14142843 & 7.14142 \\
100 & 10.04987 & 10.049875 & 10.0498756 & 10.04987 \\
500 & 22.38302 & 22.383029 & 22.3830293 & 22.38302 \\
1000 & 31.63858 & 31.638584 & 31.6385840 & 31.63858 \\
& & & & \\
\hline
\end{tabular}

Table 2: Comparison values of $-\theta^{\prime}(0) M=\zeta=\xi=\lambda=N=k_{p}=f w=h=$ $\mathrm{Nr}=\mathrm{Ec}=\mathrm{Sc}=\chi=\mathrm{Sr}=\mathbf{0}, \theta_{w}=1.0$ and $\varepsilon=1.0$.

\begin{tabular}{|l|c|c|c|c|}
\hline \multicolumn{1}{|c|}{ Pr } & Chen (1998) & $\begin{array}{c}\text { Grubka and } \\
\text { Bobba (1985) }\end{array}$ & $\begin{array}{c}\text { Dulal Pal } \\
\text { and Mondal } \\
\text { (2012) }\end{array}$ & $\begin{array}{c}\text { Present } \\
\text { results }\end{array}$ \\
\hline & & & & \\
1.0 & 1.33334 & 1.3333 & 1.33333 & 1.33231 \\
3.0 & 2.50972 & 2.5097 & 2.50971 & 2.50872 \\
7.0 & 3.97150 & --- & 3.97151 & 3.97251 \\
10 & 4.79686 & 4.7969 & 4.79687 & 4.79676 \\
100 & 15.7118 & 15.712 & --- & 15.7120 \\
\hline
\end{tabular}

\section{RESULTS AND DISCUSSIONS}

In the present analysis numerical solutions are obtained to investigate the behavior of the non- Darcy convective Casson fluid flow over elongated surface. For different critical parameters like magnetic and electrical fields, non-linear thermal radiation, Joule heating, viscous dissipation on velocity, temperature, and concentration are studied and presented through graphs using mathematical software MATLAB.

\subsection{VELOCITY PROFILES}

At higher value of the Casson parameter $\gamma$ plastic dynamic viscosity of the fluid is dominant and this leads to thinning of hydro magnetic boundary layers as a result velocity decreases. And also reduction in velocity due to suction is seen to be more than injection as shown in Fig. 2. The influence of Magnetic parameter $M$ and electric field parameter $\zeta$ on velocity can be seen in Fig. 3. Higher values of M produces Lorentz force and resists the fluid motion and reduces the velocity. It is evident that in the presence of $\zeta$ velocity distribution throughout the hydromantic boundary layer is increased. However, a little away from the stretching sheet, the enhancement in velocity is more pronounced. This shows that the streamlines are drifted away from the surface. Influence of inertial parameter $\xi$ and porosity parameter $\epsilon$ of porous medium on velocity is depicted in Fig. 4. It is clear that velocity reduces with increasing values of drag coefficient. The influence of porous permeability parameter $k_{p}$ can be observed from Fig. 5 . It is to be 
observed that that higher values of $\mathrm{k}_{\mathrm{p}}$ decreases the permeability of the porous medium as a result velocity distribution decreases in the flow. Thermal buoyancy force $\lambda$ acts as a favorable pressure gradient in accelerating the fluid flow and escalates velocity this result can be observed from the Fig. 6.The effect of solutal buoyance parameter $\mathrm{N}$ on velocity can be seen from the Fig. 7. As $\mathrm{N}$ increases velocity increase but the variation is very small. The impact of velocity slip function ' $h$ ' increases the velocity as shown in Fig. 8.

Table 3: Skin friction coefficient, Nusselt number and Sherwood number for various values of pertinent parameters when

$\theta_{w}=1.1 ; E c=0.2 ; S c=1.2 ; S r=0.2 ; \chi=0.1 ; \tau=1.1 ; \beta=0.5 ;$

$\mathrm{M}=2.0 ; \zeta=1.0 ; \xi=01 ; \lambda=0.1 ; \mathrm{N}=0.1 ; \mathrm{k}_{\mathrm{p}}=.1 ; \mathrm{f}_{\mathrm{w}}=0.1 ; \mathrm{h}=0.1 \operatorname{Pr}=2.5 ; \mathrm{Nr}=0.5$;

\begin{tabular}{|c|c|c|c|c|}
\hline $\boldsymbol{\beta}$ & $M$ & $\left(1+\frac{1}{\beta}\right) f^{\prime \prime}(0)$ & $-\left(1+N r \theta_{w}^{3}\right) \theta^{\prime}(0)$ & $-\boldsymbol{\phi}^{\prime}(\mathbf{0})$ \\
\hline $\boldsymbol{\beta}$ & $\begin{array}{l}0.5 \\
1.0 \\
1.5 \\
2.0\end{array}$ & $\begin{array}{l}-0.483353 \\
-0.416623 \\
-0.388790 \\
-0.373318\end{array}$ & $\begin{array}{l}3.171539 \\
3.172060 \\
3.171358 \\
3.170712\end{array}$ & $\begin{array}{l}1.529082 \\
1.528379 \\
1.527716 \\
1.527244\end{array}$ \\
\hline M & $\begin{array}{l}2.0 \\
3.0 \\
4.0 \\
5.0 \\
\end{array}$ & $\begin{array}{l}-0.483353 \\
-0.306358 \\
-0.211204 \\
-0.154507 \\
\end{array}$ & $\begin{array}{l}3.171539 \\
3.234910 \\
3.266369 \\
3.284047 \\
\end{array}$ & $\begin{array}{l}1.529082 \\
1.554425 \\
1.567305 \\
1.574664 \\
\end{array}$ \\
\hline$\zeta$ & \begin{tabular}{|l|}
0.01 \\
0.04 \\
0.07 \\
0.1 \\
\end{tabular} & $\begin{array}{l}-2.857979 \\
-2.782869 \\
-2.707850 \\
-2.632981 \\
\end{array}$ & $\begin{array}{l}1.656899 \\
1.753690 \\
1.841864 \\
1.923681 \\
\end{array}$ & $\begin{array}{l}1.134517 \\
1.156273 \\
1.175609 \\
1.193236 \\
\end{array}$ \\
\hline$\xi$ & $\begin{array}{l}1.0 \\
2.0 \\
3.0 \\
4.0 \\
\end{array}$ & $\begin{array}{l}-0.834979 \\
-1.133060 \\
-1.372620 \\
-1.573370 \\
\end{array}$ & $\begin{array}{l}3.025576 \\
2.881033 \\
2.751470 \\
2.633937\end{array}$ & $\begin{array}{l}1.485285 \\
1.448996 \\
1.420424 \\
1.396909 \\
\end{array}$ \\
\hline$\lambda$ & $\begin{array}{l}-0.2 \\
-0.1 \\
0.1 \\
0.2 \\
\end{array}$ & $\begin{array}{l}-0.559939 \\
-0.534089 \\
-0.482640 \\
-0.457037 \\
\end{array}$ & $\begin{array}{l}3.154186 \\
3.161174 \\
3.174882 \\
3.181601 \\
\end{array}$ & $\begin{array}{l}1.250575 \\
1.252634 \\
1.256725 \\
1.258758 \\
\end{array}$ \\
\hline $\mathrm{N}$ & \begin{tabular}{l|l|}
0.1 \\
0.2 \\
0.3 \\
0.4 \\
\end{tabular} & $\begin{array}{l}-0.482640 \\
-0.479789 \\
-0.476939 \\
-0.474090 \\
\end{array}$ & $\begin{array}{l}3.174882 \\
3.175685 \\
3.176486 \\
3.177286 \\
\end{array}$ & $\begin{array}{l}1.256725 \\
1.256969 \\
1.257213 \\
1.257457 \\
\end{array}$ \\
\hline $\mathrm{k}_{\mathrm{p}}$ & $\begin{array}{l}0.1 \\
0.5 \\
1.0 \\
1.5 \\
\end{array}$ & $\begin{array}{l}-0.483083 \\
-0.678406 \\
-0.900696 \\
-1.102258 \\
\end{array}$ & $\begin{array}{l}3.171644 \\
3.091449 \\
2.987426 \\
2.880854 \\
\end{array}$ & $\begin{array}{l}1.256588 \\
1.234410 \\
1.209180 \\
1.186342 \\
\end{array}$ \\
\hline $\mathrm{f}_{\mathrm{w}}$ & $\begin{array}{c}0.2 \\
0.1 \\
0 \\
0.1 \\
\end{array}$ & $\begin{array}{l}-0.471532 \\
-0.475317 \\
-0.479111 \\
-0.482909 \\
\end{array}$ & $\begin{array}{l}2.783772 \\
2.909286 \\
3.039651 \\
3.174778 \\
\end{array}$ & $\begin{array}{l}1.392739 \\
1.437201 \\
1.482710 \\
1.529257 \\
\end{array}$ \\
\hline $\mathrm{h}$ & $\begin{array}{l}0.1 \\
0.2 \\
0.3 \\
0.4 \\
\end{array}$ & $\begin{array}{l}-0.482909 \\
-0.366508 \\
-0.295398 \\
-0.247425 \\
\end{array}$ & $\begin{array}{l}3.174778 \\
3.145453 \\
3.126669 \\
3.113614 \\
\end{array}$ & $\begin{array}{l}1.529257 \\
1.516497 \\
1.508741 \\
1.503526 \\
\end{array}$ \\
\hline $\operatorname{Pr}$ & $\begin{array}{l}0.7 \\
1.0 \\
2.0 \\
3.0 \\
\end{array}$ & $\begin{array}{l}-0.473359 \\
-0.476029 \\
-0.481222 \\
-0.484149 \\
\end{array}$ & $\begin{array}{l}1.630610 \\
1.963624 \\
2.823630 \\
3.497811 \\
\end{array}$ & $\begin{array}{l}1.689489 \\
1.658434 \\
1.568907 \\
1.492122 \\
\end{array}$ \\
\hline $\mathrm{Nr}$ & $\begin{array}{l}0.5 \\
1.0 \\
1.5 \\
2.0 \\
\end{array}$ & $\begin{array}{l}-0.482909 \\
-0.480514 \\
-0.478666 \\
-0.477169 \\
\end{array}$ & $\begin{array}{l}3.174778 \\
3.693468 \\
4.143531 \\
4.546375 \\
\end{array}$ & $\begin{array}{l}1.529257 \\
1.589902 \\
1.625878 \\
1.649915 \\
\end{array}$ \\
\hline$\theta_{w}$ & $\begin{array}{l}1.1 \\
1.4 \\
1.7 \\
2.0 \\
\end{array}$ & $\begin{array}{l}-0.483353 \\
-0.481317 \\
-0.478965 \\
-0.476462 \\
\end{array}$ & $\begin{array}{l}3.171539 \\
3.536320 \\
3.992211 \\
4.529263 \\
\end{array}$ & $\begin{array}{l}1.529082 \\
1.613455 \\
1.677398 \\
1.720058 \\
\end{array}$ \\
\hline Ec & $\begin{array}{l}0.1 \\
0.4 \\
0.7 \\
1.0 \\
\end{array}$ & $\begin{array}{l}-0.483161 \\
-0.482411 \\
-0.481672 \\
-0.480945 \\
\end{array}$ & $\begin{array}{l}.190444 \\
3.143617 \\
3.097307 \\
3.051501 \\
\end{array}$ & $\begin{array}{l}1.528383 \\
1.531001 \\
1.533605 \\
1.536196 \\
\end{array}$ \\
\hline Sc & $\begin{array}{l}0.5 \\
1.0 \\
1.5 \\
2.0 \\
\end{array}$ & $\begin{array}{l}-0.482259 \\
-0.482729 \\
-0.482993 \\
-0.483171 \\
\end{array}$ & $\begin{array}{l}3.176426 \\
3.176236 \\
3.176136 \\
3.176074 \\
\end{array}$ & $\begin{array}{l}1.001688 \\
1.399994 \\
1.704530 \\
1.961682 \\
\end{array}$ \\
\hline$\gamma$ & $\begin{array}{l}0.1 \\
0.2 \\
0.3 \\
0.4\end{array}$ & $\begin{array}{l}-0.482909 \\
-0.482936 \\
-0.482961 \\
-0.482985\end{array}$ & $\begin{array}{l}3.174778 \\
3.174768 \\
3.174759 \\
3.174750\end{array}$ & $\begin{array}{l}1.529257 \\
1.566471 \\
1.602938 \\
1.638699\end{array}$ \\
\hline $\mathrm{Sr}$ & $\begin{array}{l}0.1 \\
0.2 \\
0.3 \\
0.4\end{array}$ & $\begin{array}{l}-0.483006 \\
-0.482909 \\
-0.482813 \\
-0.482716\end{array}$ & $\begin{array}{l}3.174742 \\
3.174778 \\
3.174813 \\
3.174849\end{array}$ & $\begin{array}{l}1.652783 \\
1.529257 \\
1.405728 \\
1.282198 \\
\end{array}$ \\
\hline
\end{tabular}

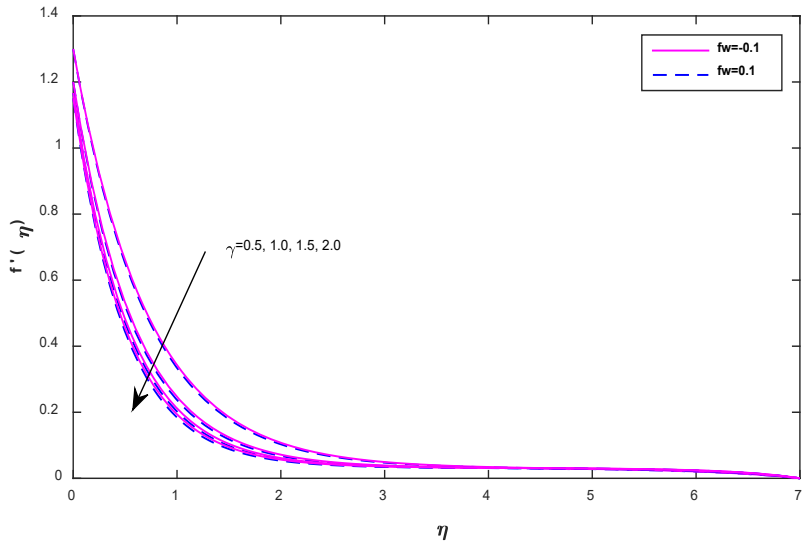

Fig. 2 Dominance of $\gamma$ on $f^{\prime}(\eta)$ for $\mathrm{fw}$

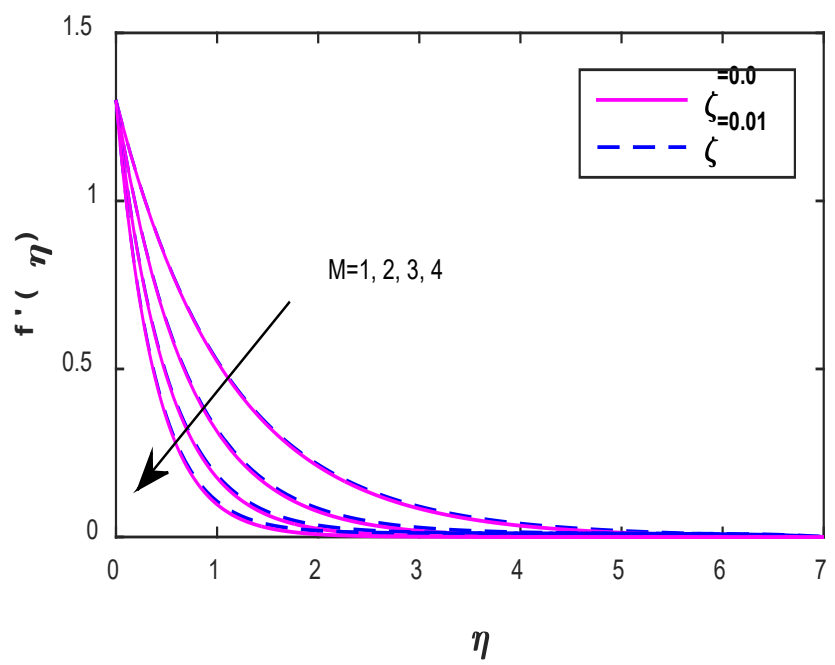

Fig. 3 Dominance of $\mathrm{M}$ on $f^{\prime}(\eta)$ for $\zeta$

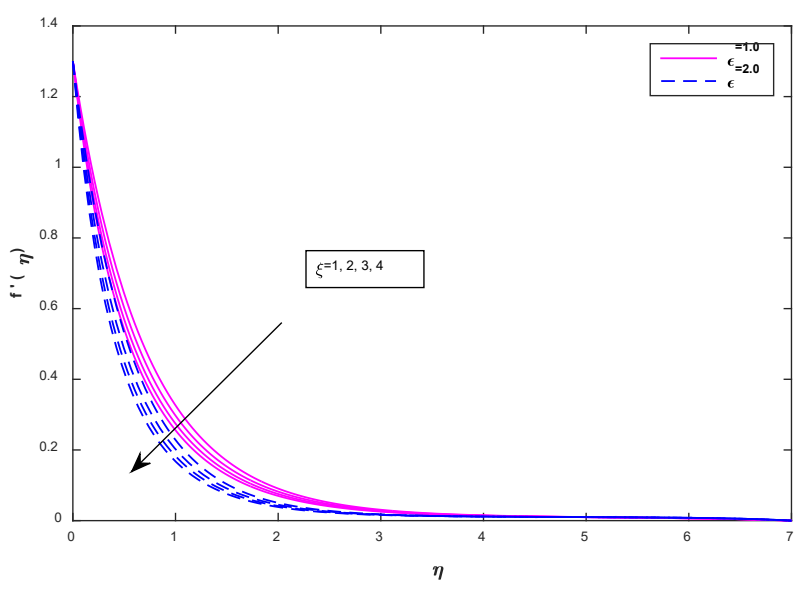

Fig. 4 Dominance of $\epsilon$ on $f^{\prime}(\eta)$ for $\xi$

\subsection{TEMPERATURE PROFILES}

It is observed from Fig. 9 that temperature is increasing function of Casson parameter $\gamma$ but it is important thing to observe that enhancement due to suction in these variables is more prominent due to injection. Influence of electric field parameter $\zeta$ can be observed from Fig.10. This $\zeta$ serves as an accelerating force to overcome the frictional resistance to increase temperature. At high Prandtl number Pr convection plays a dominant role in transforming energy from the boundary compared to conduction. It is seen from the Fig. 11 that higher Pr reduction in temperature is more rapid and also larger values of Eckert number Ec 
enhance the temperature due to release of stored energy owing to frictional heating and hence augmentation of temperature takes place. The variation of thermal radiation parameter $\mathrm{Nr}$ and temperature ratio parameter $\theta_{w}$ on temperature can be observed from Fig.12. Higher values of $\mathrm{Nr}$ corresponds to enhanced radiation which leads to higher temperatures. Increase in $\theta_{w}$ corresponds to hotter surface which in turn raises the temperature of the fluid. Increasing value of thermal buoyancy force $\lambda$ decreases temperature distribution and this can be observed from Fig. 13.

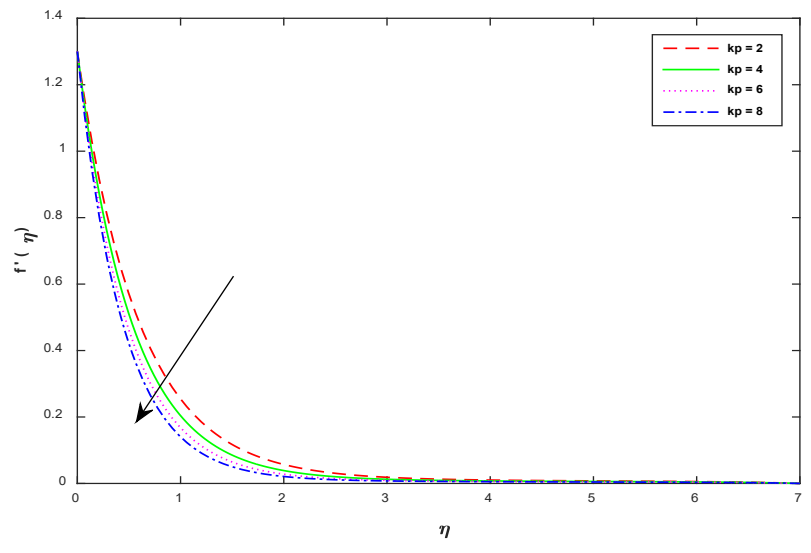

Fig. 5 Dominance of $k p$ on $f^{\prime}(\eta)$

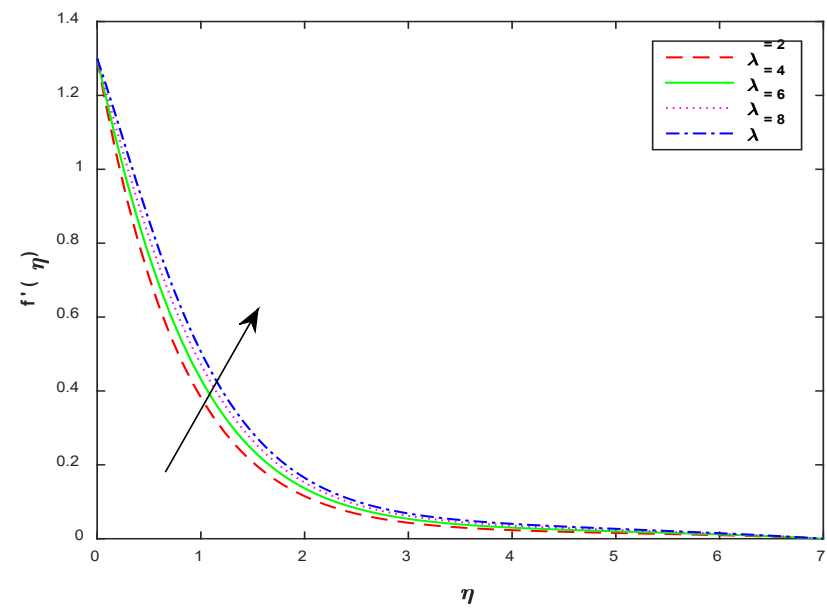

Fig. 6 Dominance of $\lambda$ on $f^{\prime}(\eta)$

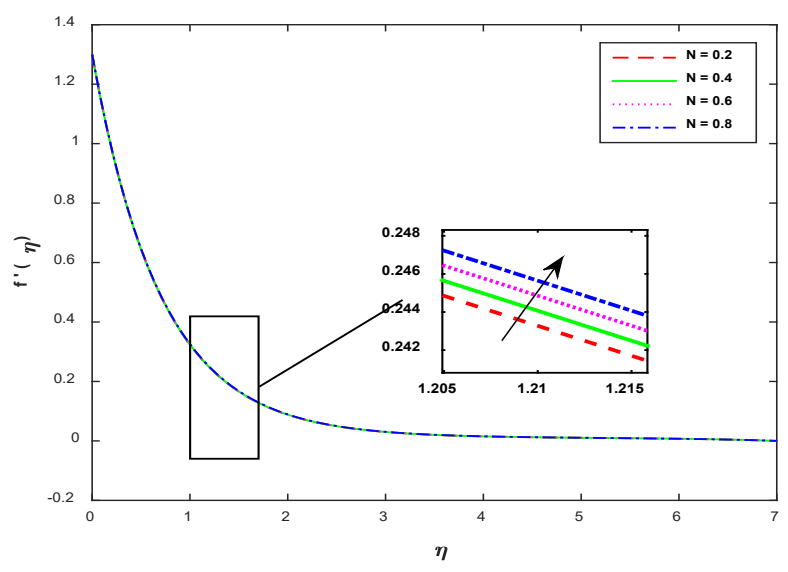

Fig. 7 Dominance of $N$ on $f^{\prime}(\eta)$

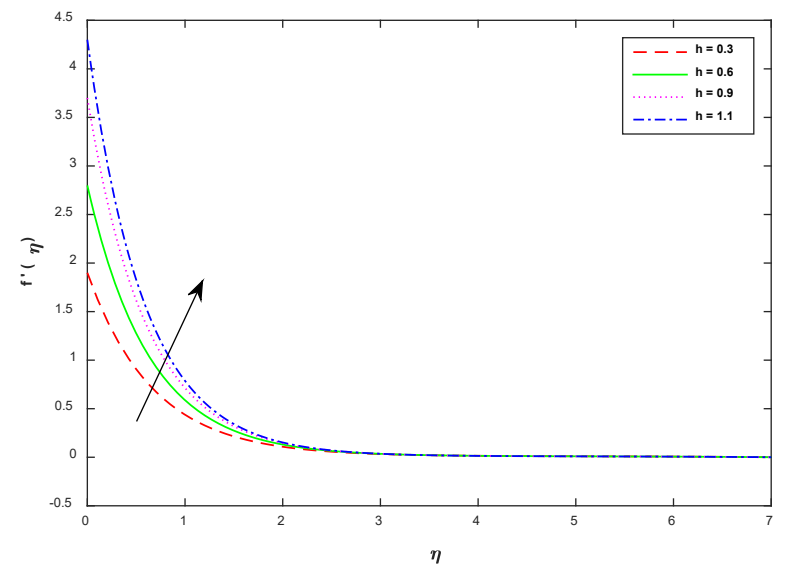

Fig. 8 Dominance of $h$ on $f^{\prime}(\eta)$

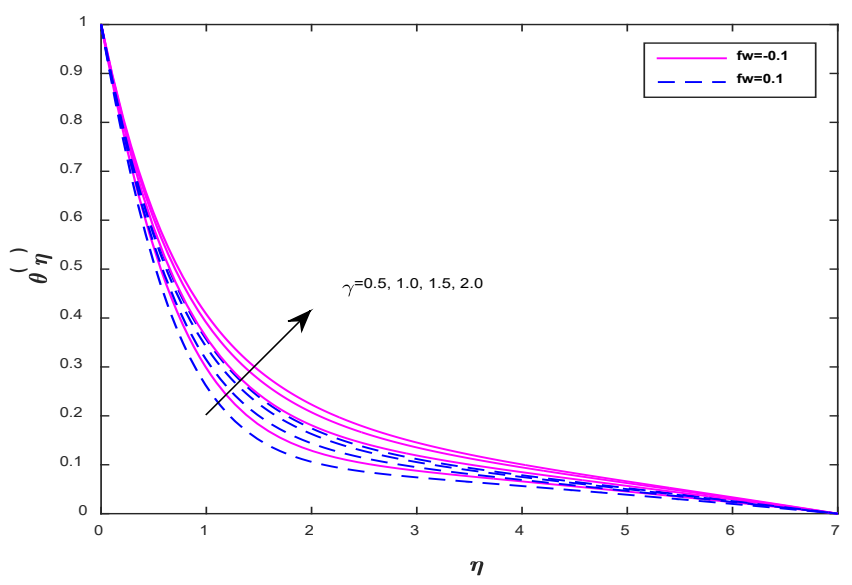

Fig. 9 Dominance of $\gamma$ on $\theta(\eta)$ for fw

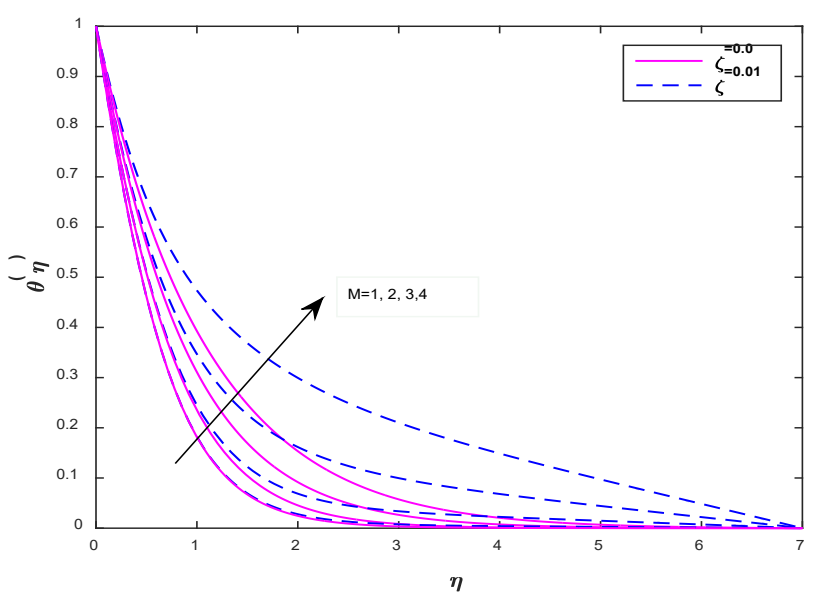

Fig. 10 Dominance of $M$ on $\theta(\eta)$ for $\zeta$

\subsection{CONCENTRATION PROFILES}

Increasing value of Casson parameter $\gamma$ increases concentration profiles by increasing thermal boundary layer thickness as shown in the Fig.14. It is observed that enhancement due to suction is more prominent than injection. Influence of electric field parameter $\zeta$ on concentration profiles for different values of magnetic parameter is clearly observed from Fig.15. Increasing value of $\zeta$ increases concentration profiles due to Lorentz force applied on it. Influence of Schmidt number $\mathrm{Sc}$ and chemical reaction $\chi$ on concentration can be observed from the Fig. 16 . It is found that the concentration from its higher value that is unity on the 
boundary begins to descend till it attains its minimum value of zero at far infinity. The concentration gets reduced for increasing value of Sc and $\chi$. This may be explained as larger values of Sc corresponds to smaller values of mass diffusivity and hence thickness of solutal boundary layer becomes thinner when $\mathrm{Sc}$ is large. This reduction due to $\chi$ is owing to the fact that destructive chemical reaction reduces the solutal boundary layer thickness and contributes for the rate of mass transfer. Soret number $\mathrm{Sr}$ have an appreciable enhancement on concentration profiles as shown in Fig.17. Variation of thermophoretic parameter $\tau$ on concentration is plotted in Fig. 18. It is observed that increasing values of thermophoretic parameter results in reduction of species concentration. Concentration of the fluid steadily changes from higher value to the lower value and ultimately fulfilling the free stream boundary condition as $\eta \rightarrow \infty$.

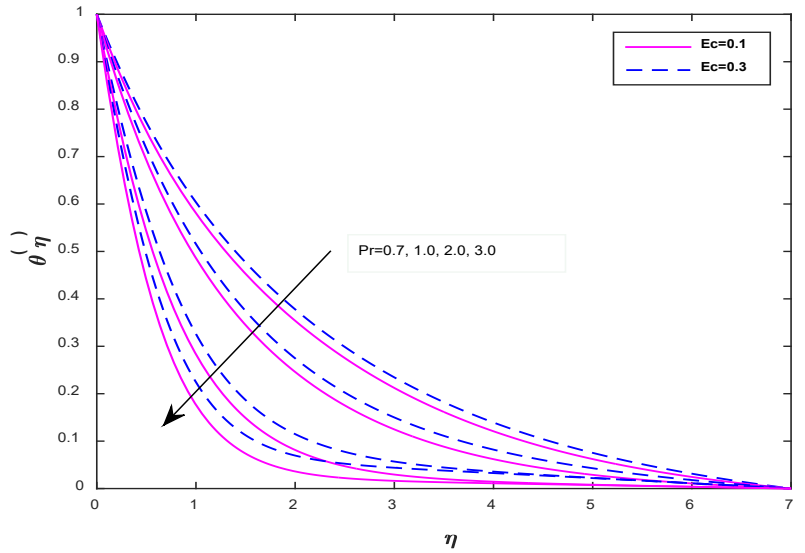

Fig. 11 Dominance of $\operatorname{Pr}$ on $\theta(\eta)$ for $E c=0.1 \& 0.3$

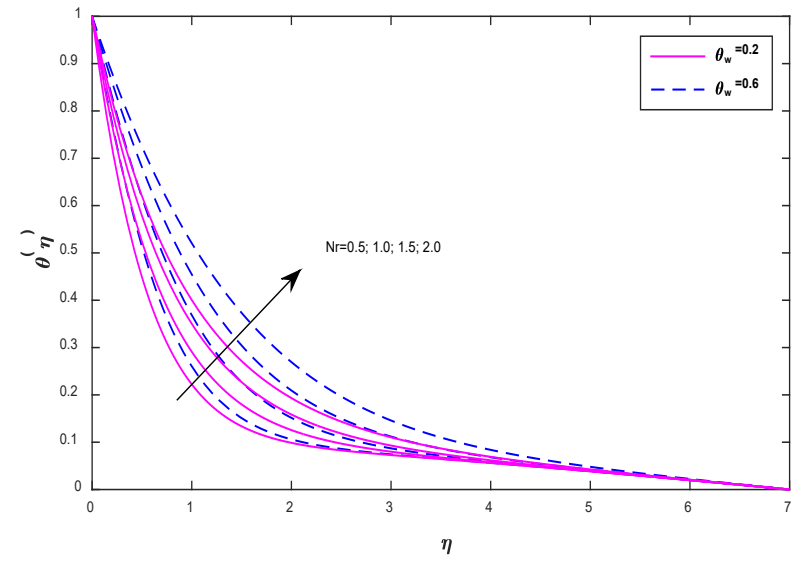

Fig. 12 Dominance of $\mathrm{Nr}$ on $\theta(\eta)$ for $\theta_{w}=0.2 \& 0.6$

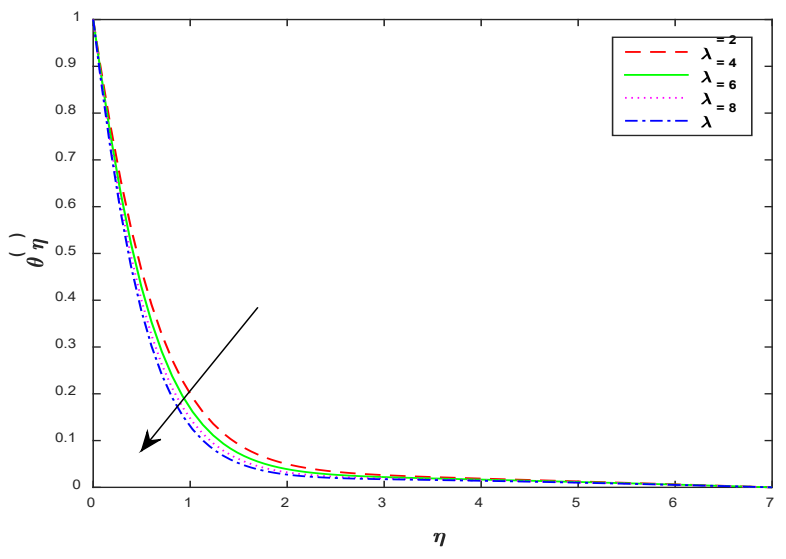

Fig. 13 Dominance of $\lambda$ on $\theta(\eta)$

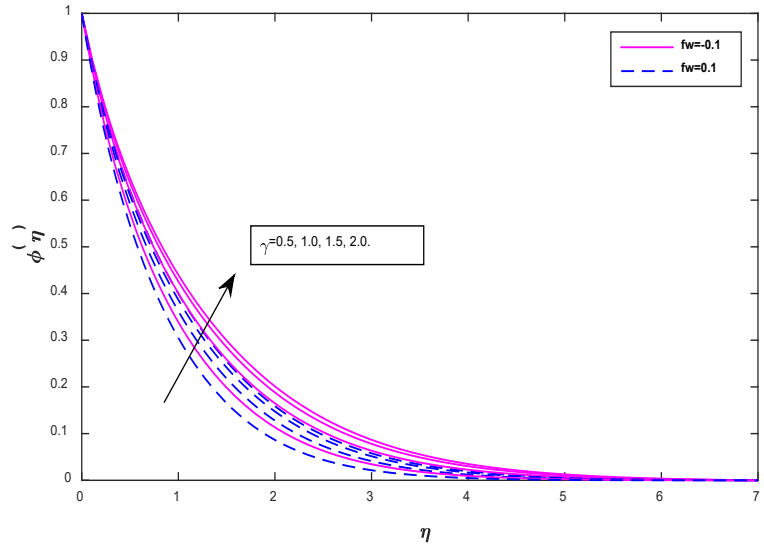

Fig. 14 Dominance of $\gamma$ on $\phi(\eta)$ for fw

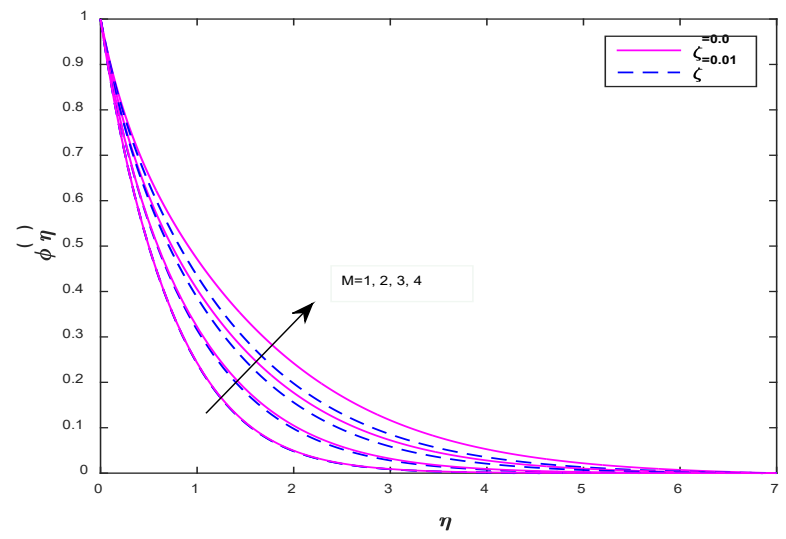

Fig. 15 Dominance of $\mathrm{M}$ on $\phi(\eta)$ for $\zeta$

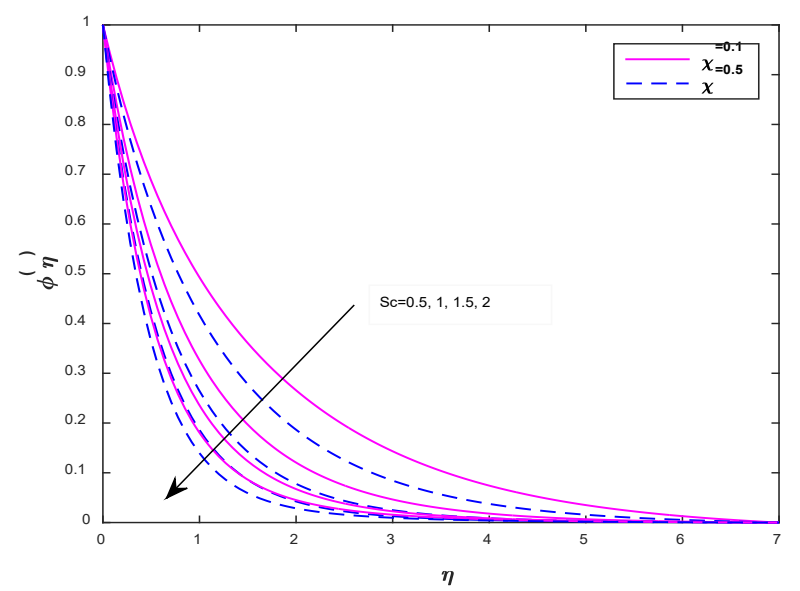

Fig. 16 Dominance of Sc on $\phi(\eta)$ for $\chi$

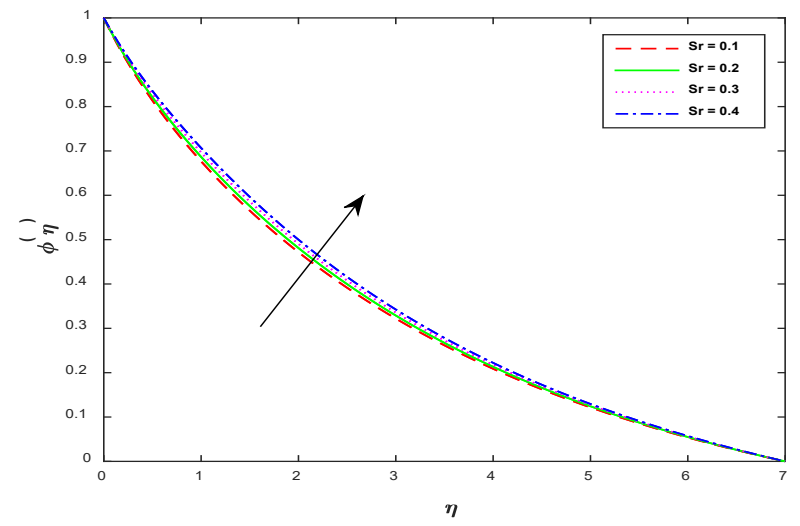

Fig. 17 Dominance of Sr on $\phi(\eta)$ 


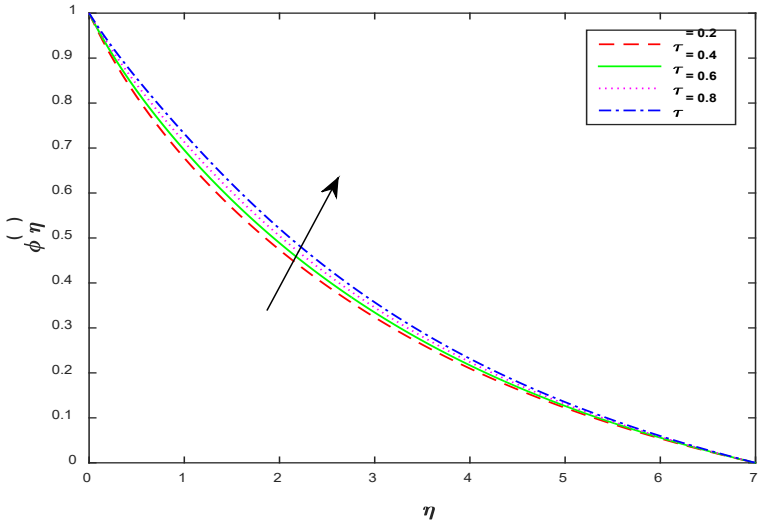

Fig. 18 Dominance of $\tau$ on $\phi(\eta)$

\section{CONCLUSIONS}

In this chapter, we studied the effect of thermo-diffusion, electrical field, non-linear thermal radiation on the mixed convective non-Darcy flow over a permeable stretching surface under the influence of a transverse magnetic field with velocity slip. The behavior of flow characteristics are analyzed and discussed in detail through the graphical plots. The significant flow features are summarized as follows.

For larger values of Casson parameter $\gamma$ the velocity decreases. Lorentz force retards the fluid flow and raises the temperature. Electrical force $\zeta$ accelerates the fluid flow and rises velocity \& thickness of the thermal boundary layer is reduced.

Inertial parameter decreased the velocity while porosity parameter $(\varepsilon)$ has an opposite effect.

Both thermal and solutal buoyancy forces $(\lambda \& N)$ are seen to enhance the velocity.

Increasing values of temperature ratio and radiation parameter ratio

$\left(\theta_{w}, N r\right)$ raise the temperature of the fluid.

Schmidt number and chemical reaction parameter $(S c, \chi)$ produce thinner solutal boundary layers with a reduction in concentration.

$>\quad$ Results of the analysis are found to be in an excellent agreement with previously published results as limiting cases.

Increasing value of slip parameter increases velocity profile.

Reduction in velocity due to suction is seen to be more than injection.

Enhancement due to suction in temparature and concentration is more prominent than due to injection.

\section{NOMENCLATURE}

$\begin{array}{cl}\bar{u}, \bar{v} & \text { Velocity components in } \mathrm{x}, \mathrm{y} \text { directions } \\ \varepsilon & \text { Porosity of the fluid } \\ v & \text { Kinematic viscosity } \\ \rho & \text { Density } \\ \mathrm{E}_{0} & \text { Electric field strength } \\ \mathrm{B}_{0} & \text { Strength of the transverse magnetic field } \\ k_{1} & \text { Porous medium permeability } \\ \mathrm{Cb}_{\mathrm{b}} & \text { Drag coefficient } \\ g^{*} & \text { Acceleration due to gravity } \\ \beta_{T} & \text { Thermal expansion coefficient } \\ \beta_{C} & \text { Concentration expansion coefficient } \\ T^{*} & \text { Inertial temperature } \\ C^{*} & \text { Fluid concentration } \\ c_{p} & \text { Specific heat at constant pressure } \\ \mathrm{k} & \text { Thermal conductivity }\end{array}$

$\begin{array}{ll}\sigma^{*} & \text { Stefen- Boltzmann constant } \\ k^{*} & \text { Absorption coefficient } \\ \mathrm{D} & \text { Mass diffusivity } \\ T_{m} & \text { Mean temperature } \\ k_{T} & \text { Thermal Diffusion Ratio } \\ k_{0} & \text { Chemical reaction of the fluid } \\ \mathrm{L} & \text { Reference length } \\ A_{0} & \text { Parameter of temperature distribution } \\ A_{1} & \text { Parameter of concentration distribution }\end{array}$

\section{REFERENCES}

Andersson, H., 1992, "MHD flow of a viscoelastic fluid past a stretching surface." Acta Mech, 95, 227-230.

https://doi.org/10.1007/BF01170814

Chamkha, A., 1997, "Hydromagnetic natural convection from anisothermal inclined surface adjacent to a thermally stratified porous medium." Int J Eng Sci, 35, 975-86.

https://doi.org/10.1016/S0020-7225 (96)00122-X

Chen, C. H., 1998, "Laminar mixed convection adjacent to vertical continuously stretching sheets," Heat and Mass Transfer, 33(5), 471476.

https://doi.org/10.1007/s00231005021

Dulal Pal and Hiranmoy Mondal., 2012, "MHD non-Darcy mixed convective diffusion of species over a stretching sheet embedded in a porous medium with non-uniform heat source/sink, variable viscosity and Soret effect,' Commun Nonlinear Sci Numer Simular, 17, 672-684. https://doi.org/10.1016/j.physb.2011.11.051

Eldabe, N. T. M., Salwa, M. G. E., 1995, "Heat transfer of MHD nonNewtonian Casson fluid flow between two rotating cylinders," J. Phys. Soc. Japan, 64, 41.

Grubka, L. J. and Bobba, K. M. ,1985, "Heat transfer characteristics of a continuous stretching surface with variable temperature, ” ASME J. Heat Transfer, 107(1), 248-250.

Gireesha, B. J., Mahanthesh, B., Manjunatha, P. T. and Gorla, R. S. R. (2015) "Numerical solution for hydromagnetic boundary layer flow and heat transfer past a stretching surface embedded in non-Darcy porous medium with fluid-particle suspension,"' J. Nigerian Mathematical Society, 34(3), 267-285.

https://doi.org/10.1016/j.jnnms.2015.07.003

Hayat, T., Hussain, Q., and Javed., T. (2009) "The modified decomposition method and pade approximations for the MHD flow over a non-linear stretching sheet," Nonlinear Anal. Real World Appl., 10, 966-973.

Khan, S. A., Saeed, T., Khan. M. I., and Hayat, T, 2019, "Entropy optimized CNTs based Darcy-Forchheimer nanomaterial flow between two stretchable rotating disks,"International Journal of Hydrogen

Energy,

https://doi: 10.1016/j.ijhydene.2019.10.053.

Kolli Vijaya and Gurrampti Venkata Ramana Reddy, 2019, "Magnetohydrodynamic Casson Fluid Flow Over a Vertical Porous Plate in the Presence of Radiation, Soret and Chemical Reaction Effects

Journal of Nanofluids, Volume 8, Number 6, pp. 1240-1248(9). https://doi.org/10.1166/jon.2019.1684 
Mabood, F., and Das, K., 2016, "Melting heat transfer on hydromagnetic flow of a nanofluid over a stretching sheet with radiation and second order slip," European Phys. J. Plus, 131(3), 1-12.

Mabood, F., Imtiaz, M., Alsaedi, A. and Hayat, T., 2016, "Unsteady convective boundary layer flow of Maxwell fluid with nonlinear thermal radiation A numerical study," Int. J. Nonlinear Sciences and Numerical Simulation, 1-9.

Narayana, M., Khidir, A. A., Sibanda, P., and Murthy, P. V. S. N., 2013, "Soret effect on the natural convection from a vertical plate in a thermally stratified porous medium saturated with non-Newtonian liquid," Journal of Heat Transfer, 135(3), 1-10.

https://doi.org/10.1115/1.4007880

Partha, M. K., 2009, "Suction/injection effects on thermophoresis particle deposition in a non-Darcy porous medium under the influence of Soret, Dufour effects," Int. J. Heat and Mass Transfer, 52, 1971-1979. https://doi.org/10.1016/j.ijheatmasstransfer.2008.07.055

Pavlov, K., 1974, "Magneto hydrodynamic flow of an incompressible viscous fluid caused by the deformation of a plane surface." Magnetohydrodynamic,10, 146-148.

http://doi.org/10.22364/mhd

Rahmat Ellahi., Sadiq M. Sait., Shezad, N., Mobin, 2019, "Numerical simulation and mathematical modeling of electroosmotic CouettePoiseuille flow of MHD power-law nanofluid with entropy generation," Symmetry, 11(8), 1038.

https://doi.org/10.3390/sym11081038

Ramana Reddy, G. V., Bhaskar Reddy, N., \& Chamkha, A. J., 2016, "MHD mixed convection oscillatory flow over a vertical surface in a porous medium with chemical reaction and thermal radiation," Journal of Applied Fluid Mechanics, 9(3), 1221-1229.
Ramana Reddy, G. V., Bhaskar Reddy, N., \& Gorla, R. S. R., 2016, "Radiation and chemical reaction effects on MHD flow along a moving vertical porous plate," International Journal of Applied Mechanics and Engineering, 21(1), 157-168.

http://dx.doi.org/doi:10.1515/ijame-2016-0010.

Reddy, G. V. R., \& Chamkha, A. J., 2015, "Lie group analysis of chemical reaction effects on MHD free convection dissipative fluid flow past an inclined porous surface," International Journal of Numerical Methods for Heat and Fluid Flow, 25(7), 1557-1573.

http://dx.doi.org/ 10.1108/HFF-08-2014-0270

Swati Mukopadhyaya and Vajravelu, K. 2013, "Diffusion of chemically reactive species in Casson fluid flow over an unsteady permeable stretching surface," Journal of Hydrodynamics, 25(4), 591-598.

https://doi.org/10.1016/S1001-6058 (11)60400-X

Vempati, S. R., and Laxmi-Narayana-Gari, A. B., 2010, "Soret and Dufour effects on unsteady MHD flow past an infinite vertical porous plate with thermal radiation," Appl. Math. Mech. Engl. Ed, 31(12), 1481-1496.

https://doi.org/10.1007/s10483-010-1378-9.

Vijaya, N., Hari Krishna, Y., Kalyani, K., \& Reddy, G. V. R., 2018, "Soret and radiation effects on an unsteady flow of a casson fluid through porous vertical channel with expansion and contraction", Frontiers in Heat and Mass Transfer, 11. http://dx.doi.org/10.5098/hmt.11.19

Nagalakshmi, P. S, S., Vijaya, N., 2020, "MHD flow of Carreau Nanofluid explored using CNT over a nonlinear stretched sheet," Frontiers in Heat and Mass Transfer (FHMT), 14, 4. http://dx.doi.org/doi: 10.5098/hmt.14.4

Zeeshan, A. and Majeed, A., 2016, "Non-Darcy Mixed Convection Flow of Magnetic Fluid over a Permeable Stretching Sheet with Ohmic Dissipation,' Journal of Magnetics, 21(1), 153-158.

https://doi.org/10.4283/JMAG.2016.21. 